\title{
Different anti-remodeling effect of nilotinib and fluticasone in a chronic asthma model
}

\author{
Hye Seon Kang ${ }^{1,{ }^{*}}$, Chin Kook Rhee ${ }^{1,{ }^{\star}}$, Hea Yon Lee ${ }^{1}$, Hyoung Kyu Yoon ${ }^{2}$, Soon Seok Kwon ${ }^{3}$, \\ and Sook Young Lee ${ }^{1}$
}

\begin{abstract}
${ }^{1}$ Division of Pulmonary, Allergy and Critical Care Medicine, Department of Internal Medicine, College of Medicine, Seoul St. Mary's Hospital, The Catholic University of Korea, Seoul; '²Division of Pulmonary, Allergy and Critical Care Medicine, Department of Internal Medicine, College of Medicine, Yeouido St. Mary's Hospital, The Catholic University of Korea, Seoul; ' 3 Division of Pulmonary, Allergy and Critical Care Medicine, Department of Internal Medicine, College of Medicine, Bucheon St. Mary's Hospital, The Catholic University of Korea, Bucheon, Korea
\end{abstract}

Received: January 4, 2015

Revised : July 5, 2015

Accepted: August 16, 2015

\section{Correspondence to}

Sook Young Lee, M.D.

Division of Pulmonary, Allergy

and Critical Care Medicine,

Department of Internal Medicine, College of Medicine, Seoul St.

Mary's Hospital, The Catholic

University of Korea, 222 Ban-

po-daero, Seocho-gu, Seoul o6591,

Korea

Tel: +82-2-2258-6061

Fax: +82-2-596-2158

E-mail: sooklee@catholic.ac.kr

*These authors contributed equally to this work.
Background/Aims: Inhaled corticosteroids are the most effective treatment currently available for asthma, but their beneficial effect against airway remodeling is limited. The tyrosine kinase inhibitor nilotinib has inhibitory activity against c-kit and the platelet-derived growth factor receptor. We compared the effects of fluticasone and nilotinib on airway remodeling in a chronic asthma model. We also examined whether co-treatment with nilotinib and fluticasone had any synergistic effect in preventing airway remodeling.

Methods: We developed a mouse model of airway remodeling, including smooth muscle thickening, in which ovalbumin (OVA)-sensitized female BALB/c-mice were repeatedly exposed to intranasal OVA administration twice per week for 3 months. Mice were treated with fluticasone and/or nilotinib intranasally during the OVA challenge.

Results: Mice chronically exposed to OVA developed eosinophilic airway inflammation and showed features of airway remodeling, including thickening of the peribronchial smooth muscle layer. Both fluticasone and nilotinib attenuated airway smooth muscle thickening. However, only nilotinib suppressed fibrotic changes, demonstrating inhibition of collagen deposition. Fluticasone reduced pro-inflammatory cells, such as eosinophils, and several cytokines, such as interleukin 4 (IL-4), IL-5, and IL-13, induced by repeated OVA challenges. On the other hand, nilotinib reduced transforming growth factor $\beta 1$ levels in bronchoalveolar lavage fluid and inhibited fibroblast proliferation significantly.

Conclusions: These results suggest that fluticasone and nilotinib suppressed airway remodeling in this chronic asthma model through anti-inflammatory and anti-fibrotic pathways, respectively.

Keywords: Asthm; Nilotinib; Fluticasone; Airway remodeling

\section{INTRODUCTION}

Asthma is characterized by inflammation of the airways, reversible airflow obstruction, and airway hyper-respon- siveness. Airway remodeling can be defined as changes in the composition and organization of the cells and extracellular constituents of the airway wall [1]. These structural changes increase airway wall thickness, result- 
ing in a decline in lung function and resistance to asthma treatment [2]. Airway remodeling is characterized by subepithelial fibrosis, increased airway smooth muscle mass, goblet cell hyperplasia, and angiogenesis [3].

Inhaled corticosteroids remain the mainstay of anti-inflammatory treatment in asthma. Many studies have investigated the effects of inhaled corticosteroids on airway remodeling. Chetta et al. [4] reported that high doses of fluticasone affected airway remodeling by reducing both submucosal vascularity and basement membrane thickness. It has been demonstrated that inhaled corticosteroids prevent airway remodeling through inhibition of thickening of the airway smooth muscle layer [5-7]. However, the effect of inhaled corticosteroids on subepithelial fibrosis is controversial $[8,9]$.

We previously reported that a tyrosine kinase inhibitor can ameliorate both airway inflammation and airway remodeling in a chronic asthma model $[10,11]$. Nilotinib (Tasigna, Novartis Pharmaceuticals, Basel, Switzerland) is a small-molecule tyrosine kinase inhibitor developed to treat chronic myeloid leukemia [12]. Nilotinib inhibits the tyrosine kinase activity of not only the Abl kinases but also c-kit and the platelet-derived growth factor (PDGF) receptor [12]. Additionally, nilotinib targets transforming growth factor $\beta$ (TGF- $\beta$ ), a profibrotic cytokine thought to play important roles in fibrotic tissue remodeling in asthma, and has been investigated in many fibrotic diseases [13-16].

The aim of this study was to compare the effects of fluticasone and nilotinib on airway remodeling in a chronic asthma model. We also examined whether co-treatment with nilotinib and fluticasone had any synergistic effect in the prevention of airway remodeling.

\section{METHODS}

\section{Sensitization and antigen challenge protocol}

Female 6-week-old BALB/c mice weighing 20 to $25 \mathrm{~g}$ were used ( $\mathrm{n}=7$ to 9 mice per group; Orient Bio Experimental Animal Center, Seongnam, Korea). Mice were assigned to one of the following five treatment groups: (1) control; (2) ovalbumin (OVA, grade V; Sigma-Aldrich, St. Louis, MO, USA) challenge; (3) OVA challenge plus $100 \mu \mathrm{g} / \mathrm{kg}$ fluticasone; (4) OVA challenge plus $80 \mathrm{mg} /$ kg nilotinib; or (5) OVA challenge plus $100 \mu \mathrm{g} / \mathrm{kg}$ fluti- casone and $80 \mathrm{mg} / \mathrm{kg}$ nilotinib. Mice were immunized by subcutaneous injection on days $0,7,14$, and 21 with $25 \mu \mathrm{g}$ of OVA absorbed to $1 \mathrm{mg}$ of aluminum hydroxide (Sigma-Aldrich, Milwaukee, WI, USA) in $200 \mu \mathrm{L}$ of phosphate-buffered saline (PBS). Intranasal OVA challenges (20 $\mu \mathrm{g} / 50 \mu \mathrm{L}$ in PBS) were administered on days 27, 29, and 31 under isoflurane (Vedco, St. Joseph, MO, USA) anesthesia. Intranasal OVA challenges were then repeated twice per week for 3 months. Allergen-sensitized mice were treated with fluticasone by intranasal administration and/or nilotinib by oral gavage. Fluticasone and/or nilotinib were given five times per week starting on day 35 and then during the OVA challenges for 3 months. Mice were sacrificed 24 hours after the final OVA challenge, and lung tissues were analyzed. All animal experimental protocols were approved by The Catholic University of Korea, Animal Subjects Committee.

\section{Bronchoalveolar lavage}

Mice were sacrificed by $\mathrm{CO}_{2}$ asphyxiation. The trachea was exposed and cannulated with silicone tubing attached to a 23-gauge needle on an 800- $\mu \mathrm{L}$ tuberculin syringe. After instillation of $1 \mathrm{~mL}$ sterile PBS through the trachea into the lung, BAL fluid was withdrawn. The total number of cells in the BAL fluid was counted using a hemocytometer. The BAL fluid was cytospun (7 minutes, 2,000 rpm) onto microscope slides and stained with Diff-Quick (Sysmax, Kobe, Japan). The percentages of macrophages, eosinophils, lymphocytes, and neutrophils in the BAL fluid were obtained by counting 400 leukocytes on randomly selected portions of the slides using light microscopy. Supernatants were stored at $-70^{\circ} \mathrm{C}$ until the day of measurement.

\section{Enzyme-linked immunosorbent assay}

The concentrations of interleukin 4 (IL-4), IL-5, IL-13, and TGF- $\beta 1$ were measured in the BAL fluid with enzyme-linked immunosorbent assay (ELISA) kits (R\&D Systems, Minneapolis, MN, USA). The acid-activated latent form of TGF- $\beta 1$ was measured. The protocol followed was according to the manufacturers' instructions. The sensitivity of the ELISA was as follows: (1) IL-4, 2 $\mathrm{pg} / \mathrm{mL}$; (2) IL-5, $7 \mathrm{pg} / \mathrm{mL}$; (3) IL-13, $1.5 \mathrm{pg} / \mathrm{mL}$; and (4) TGF- $\beta 1,15.6 \mathrm{pg} / \mathrm{mL}$. 


\section{Immunohistochemistry}

Six-micron-thick sections of lung from each paraffin block were deparaffinized with xylene and rehydrated in an ethanol series. For immunohistochemical detection of $\alpha$-smooth muscle actin, the lung sections were incubated overnight at $4^{\circ} \mathrm{C}$ with a primary monoclonal antibody against $\alpha$-smooth muscle actin (Sigma-Aldrich) or mouse serum as a negative control instead of the primary antibody. Immunoreactivity was detected by sequential incubations of lung sections with a biotinylated secondary antibody, followed by peroxidase reagent and the 3-amino-9-ethylcarbazole (AEC) chromogen. The immunostaining area of $\alpha$-smooth muscle actin in each paraffin wax-embedded lung was outlined and quantified using a light microscope attached to an image analysis system (BX50, Olympus, Tokyo, Japan). Results are expressed as the area of immunostaining per micron length of basement membrane of bronchioles (internal diameter, 150 to $200 \mu \mathrm{m}$ ). At least 10 bronchioles were counted on each slide.

\section{Hydroxyproline analysis}

Aliquots of lung tissue (6o mg) from each mouse were used for the hydroxyproline assays. A sample of lung homogenate was added to $250 \mu \mathrm{L}$ of $12 \mathrm{~N} \mathrm{HCl}$ for 16 hours at $110^{\circ} \mathrm{C}$. Following centrifugation, $25 \mu \mathrm{L}$ of each supernatant was assayed. A total of $25 \mu \mathrm{L}$ of citrate/acetate buffer (5\% citric acid, $7.2 \%$ sodium acetate, $3.4 \%$ sodium hydroxide, and $1.2 \%$ glacial acetic acid) and $500 \mu \mathrm{L}$ of chloramine-T solution (1.41 g chloramine-T, $26 \mathrm{~mL}$ n-propanol, 20.7 $\mathrm{mL}$ distilled water, and $53.3 \mathrm{~mL}$ citrate/ acetate buffer) were added to a $25 \mu \mathrm{L}$ sample of the digested lung. The resulting samples were then incubated at room temperature for 20 minutes before $500 \mu \mathrm{L}$ of Ehrlich's solution (4.5 g $\rho$-dimethylaminobenzaldehyde, $18.6 \mathrm{~mL} n$-propanol, and $7.8 \mathrm{~mL} 70 \%$ perchloric acid) was added. These samples were incubated for $15 \mathrm{~min}$ utes at $65^{\circ} \mathrm{C}$, and cooled samples were read at $550 \mathrm{~nm}$ in a spectrophotometer. Hydroxyproline concentrations were calculated from a standard curve.

\section{Measurement of smooth muscle area}

The immunostained area of $\alpha$-smooth muscle actin in each paraffin wax-embedded lung was outlined and quantified using a light microscope attached to an image analysis system (BX50, Olympus). Results are expressed as the area immunostained per micrometer length of basement membrane of bronchioles (internal diameter, 650 to $750 \mu \mathrm{m}$ ). At least 10 bronchioles were counted on each slide.

\section{Proliferation assay}

Cell proliferation was quantified by the colorimetric XTT-based assay kit (Boehringer Mannheim, Mannheim, Germany). A lung fibroblast cell line (HFL-1) was obtained from the American Type Culture Collection (No. CCL-153, Rockville, MD, USA). Cells were seeded in 96-well microtiter plates (Greiner, Frickenhausen, Germany) at a concentration of $5 \times 10^{3}$ cells $/ \mathrm{mL}$. Cells were incubated in Dulbecco's modified Eagle's medium containing $10 \%$ fetal bovine serum and $1 \%$ penicillin-streptomycin at $37^{\circ} \mathrm{C}$ in a humidified atmosphere of $5 \% \mathrm{CO}_{2}$ for 24 hours, then serum-starved for 24 hours. Nilotinib or fluticasone was co-administered with PDGF-AA or stem cell factor (SCF), and cells were incubated for 72 hours. Next, $50 \mu \mathrm{L}$ of XT'T solution was added for 4 hours at $37^{\circ} \mathrm{C}$. Absorbance was measured at $450 \mathrm{~nm}$ in a Dynatech MR 3.13 MicroELISA reader (Dynex Technologies, Ashford, UK).

\section{Data analysis}

Results from each group were compared with Student $t$ test. All statistical analyses were performed using SPSS software version 18.o (SPSS Inc., Chicago, IL, USA). A value of $p<0.05$ was considered to indicate statistical significance. All results are given as mean $\pm \mathrm{SE}$.

\section{RESULTS}

\section{Effects of fluticasone and nilotinib on airway inflam- mation}

Repeated OVA challenge induced a significant increase in the number of total cells and eosinophils in the BAL fluid. Fluticasone treatment in OVA-challenged mice reduced significantly the numbers of total cells, eosinophils, and neutrophils in BAL fluid (Fig. 1). There was no significant difference in the numbers of total cells, macrophages, eosinophils, lymphocytes, or neutrophils between the OVA and nilotinib group. The combination of fluticasone and nilotinib treatment reduced only the number of eosinophils. 




Figure 1. Effects of fluticasone and/or nilotinib on total and differential cell counts in bronchoalveolar lavage fluid (BALF). OVA, ovalbumin; AF, fluticasone; AN, nilotinib; ANF, fluticasone plus nilotinib. ${ }^{a} p<0.01$ for control vs. OVA, ${ }^{b} p<0.01,{ }^{c} p<0.05$ for OVA vs. AF or OVA vs. AN or OVA vs. ANF.

\section{Effects of fluticasone and nilotinib on lung histopa- thology}

Histological lung sections demonstrated that repeated OVA challenge induced marked increases in subepithelial, peribronchial, and perivascular inflammation compared with the control group. In sensitized mice, the airway architecture was distorted by epithelial folding, subepithelial fibrosis, and lumen narrowing compared with control mice. These histopathological inflammatory changes were reduced after treatment with fluticasone and nilotinib, and co-administration of both drugs showed synergistic effects (Fig. 2).

\section{Effects of fluticasone and nilotinib on the area of the airway smooth muscle layer}

Repeated OVA challenge resulted in a significant increase in the area of peribronchial $\alpha$-smooth muscle actin immunostaining (control, $0.57 \pm 0.06 \mu \mathrm{m}^{2} / \mu \mathrm{m}$ vs. OVA, $1.80 \pm 0.67 \mu \mathrm{m}^{2} / \mu \mathrm{m}$; circumference of bronchiole, respectively; $p<0.01)$. Fluticasone significantly reduced the area of peribronchial $\alpha$-smooth muscle actin staining in mice subjected to repeated OVA challenge (fluticasone, $0.10 \pm 0.30$ vs. OVA, $1.80 \pm 0.67$, respectively; $p<$ o.o1). Nilotinib and the combination of nilotinib and fluticasone also significantly reduced the area of peribronchial $\alpha$-smooth muscle actin staining in repeatedly OVA-challenged mice (nilotinib, $1.35 \pm 0.59$ vs. OVA, 1.80
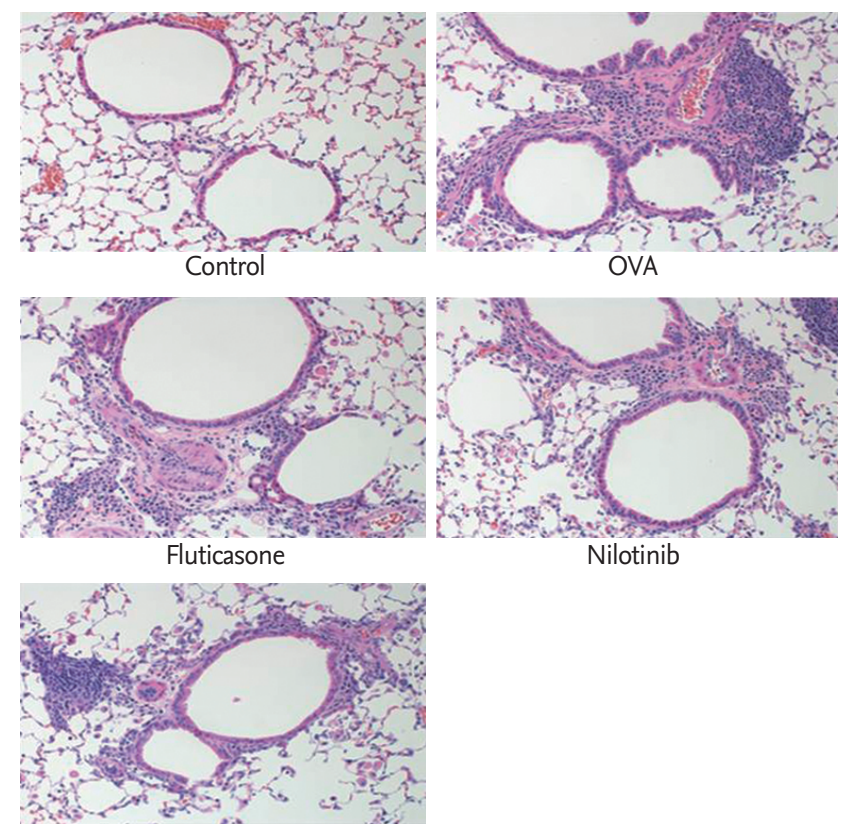

Fluticasone + nilotinilb

Figure 2. Effects of fluticasone and/or nilotinib on the histopathological changes in a chronic asthma lung model. Representative images of lung sections from each group (H\&E stain, $\times 200$ ). Repeated ovalbumin (OVA) challenges induced marked increases in subepithelial, peribronchial, and perivascular inflammation. These changes were attenuated by treatment with fluticasone or nilotinib.

\pm 0.67 , respectively; nilotinib + fluticasone, $1.29 \pm 0.53 \mathrm{vs}$. OVA, $1.80 \pm 0.67$, respectively; $p<0.05$ for both) (Fig. 3 ).

\section{Effects of fluticasone and nilotinib on IL-4, IL -5 , and IL -13 levels}

Repeated OVA challenges induced significant increases in the levels of IL-4, IL-5, and IL-13 in BAL fluid. Fluticasone treatment significantly reduced the levels of IL-4 (119.34 \pm 51.87 vs. $60.31 \pm 14.31, p<0.01)$, IL-5 (49.34 \pm 16.79 vs. $26.49 \pm 19.35, p<0.05)$, and IL-13 (88.37 \pm 33.54 vs. $37.07 \pm 17.25, p<0.01)$. Nilotinib treatment significantly reduced the levels of IL-4 (119.34 \pm 51.87 vs. $72.76 \pm 18.69$, $p<0.05)$ and IL-13 (88.37 \pm 33.54 vs. $49.20 \pm 33.80, p<0.05)$. Co-treatment with nilotinib and fluticasone significantly reduced the IL-5 level (49.34 \pm 16.79 vs. $30.99 \pm 18.89$, p $<0.05)$ (Fig. 4).

\section{Effects of fluticasone and nilotinib on collagen levels}

Collagen deposition was assessed by measuring the hydroxyproline content in lung tissues. The level of hy- 

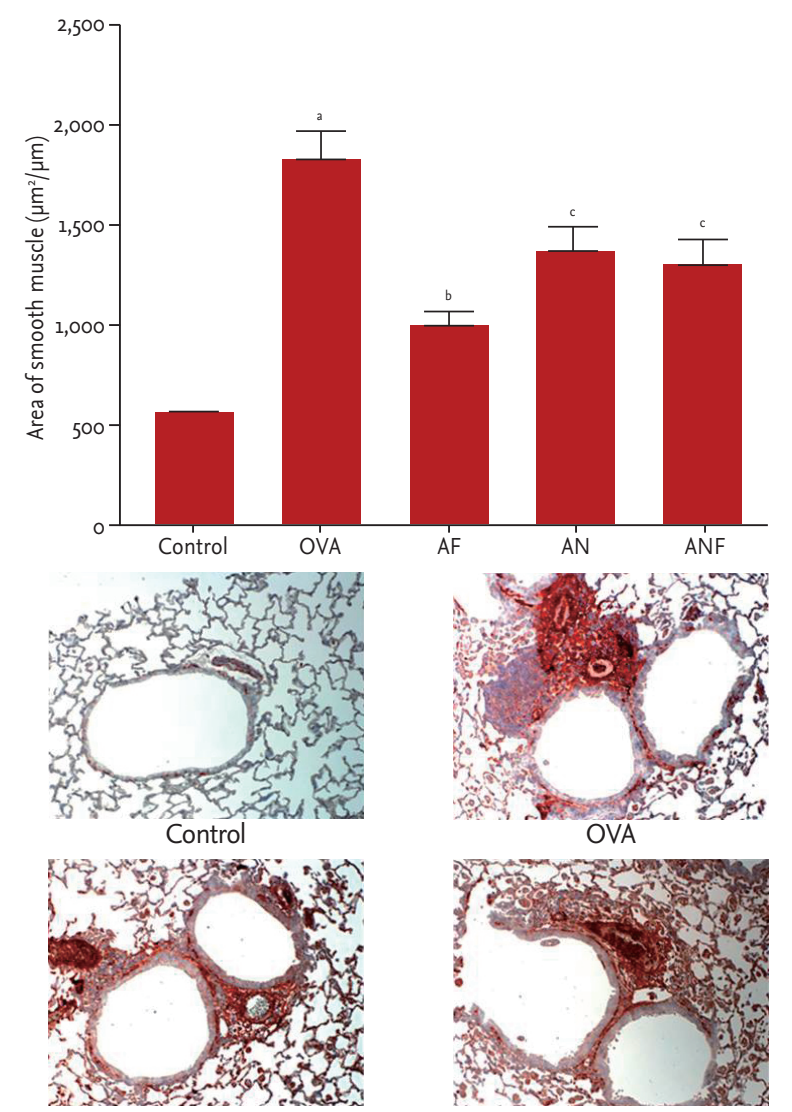

Fluticasone
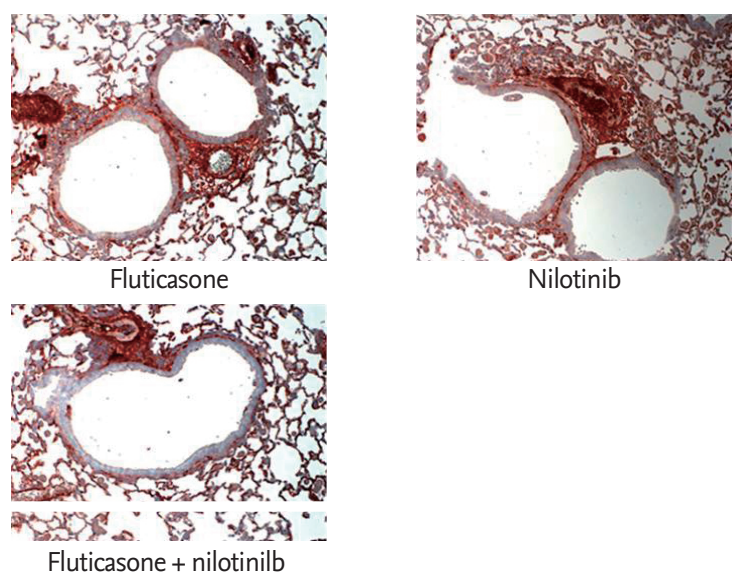

Nilotinib

Figure 3. Effects of nilotinib and/or fluticasone on the area of the airway smooth muscle layer. Representative histological lung sections (×200) from each group. The smooth muscle area was calculated as the immunostained area per micron length of the basement membrane of bronchioles using an image analyzer. Values are mean $\pm \mathrm{SE}(\mathrm{n}=7$ to 18 per group). Repeated ovalbumin (OVA) challenges induced an increase in the immunostaining area, which was inhibited by fluticasone and/or nilotinib treatment. AF, fluticasone; AN, nilotinib; ANF, fluticasone plus nilotinib. ${ }^{a} p<0.01$ compared with the control group, ${ }^{b} p<0.01,{ }^{c} p<0.05$ compared with the OVA group.

droxyproline was measured in $\mu \mathrm{g} / \mathrm{lung}$. Repeated OVA challenges induced a significant increase in the level of hydroxyproline (control, $2.75 \pm 1.54$ vs. OVA, $7.16 \pm 2.14$, respectively; $p<0.01$ ). Nilotinib treatment significantly reduced the level of hydroxyproline (nilotinib, $4.78 \pm 1.13$

vs. OVA, 7.16 \pm 2.14 , respectively; $p<0.050$ ) (Fig. 5). The level of hydroxyproline showed a trend towards reduction with fluticasone and combined fluticasone and nilotinib treatment.

\section{Effects of fluticasone and nilotinib on TGF- $\beta 1$ levels} Repeated OVA challenges induced a significant increase in the level of TGF- $\beta 1$ (control, $659.46 \pm 276.84$ vs. OVA, $1661.34 \pm 408.58$, respectively; $p<0.01$ ). Nilotinib treatment significantly reduced the level of TGF- $\beta 1$ (nilotinib, $1,070.66 \pm 239.58$ vs. OVA, $1,661.34 \pm 408.58$, respectively; $p<0.01$ ) (Fig. 6). However, neither fluticasone alone nor co-administration with nilotinib reduced the level of TGF- $\beta 1$.

\section{Effects of fluticasone and nilotinib on growth of lung fibroblasts stimulated by PDGF and SCF}

Nilotinib and the combination of nilotinib and fluticasone inhibited PDGF-AA-stimulated cell proliferation. However, fluticasone alone had no inhibitory effect on PDGF-AA-stimulated cell proliferation. The addition of fluticasone and/or nilotinib significantly inhibited SCF-induced cell proliferation (Fig. 7).

\section{DISCUSSION}

In this study, we demonstrated that both fluticasone and nilotinib treatment can attenuate airway smooth muscle thickening. However, only nilotinib suppressed fibrotic changes, demonstrating inhibition of collagen deposition as measured by hydroxyproline analysis. Pro-inflammatory cells, such as eosinophils, and several cytokines, such as IL-4, IL-5, and IL-13, induced by repeated OVA challenge, were reduced by fluticasone. We confirmed that fluticasone had anti-inflammatory effects while nilotinib was more effective in reducing the levels of TGF- $\beta 1$. Thus, we suggest that fluticasone and nilotinib have therapeutic effects on airway smooth muscle remodeling in different ways.

Inhaled corticosteroids are the mainstay of anti-inflammatory treatment in asthma, but their effect on airway fibrosis is debatable [8,9]. This may be because airway remodeling in asthma is a consequence of mechanical stimuli as well as inflammation. The response of airway epithelial cells to acute compressive stress during bron- 

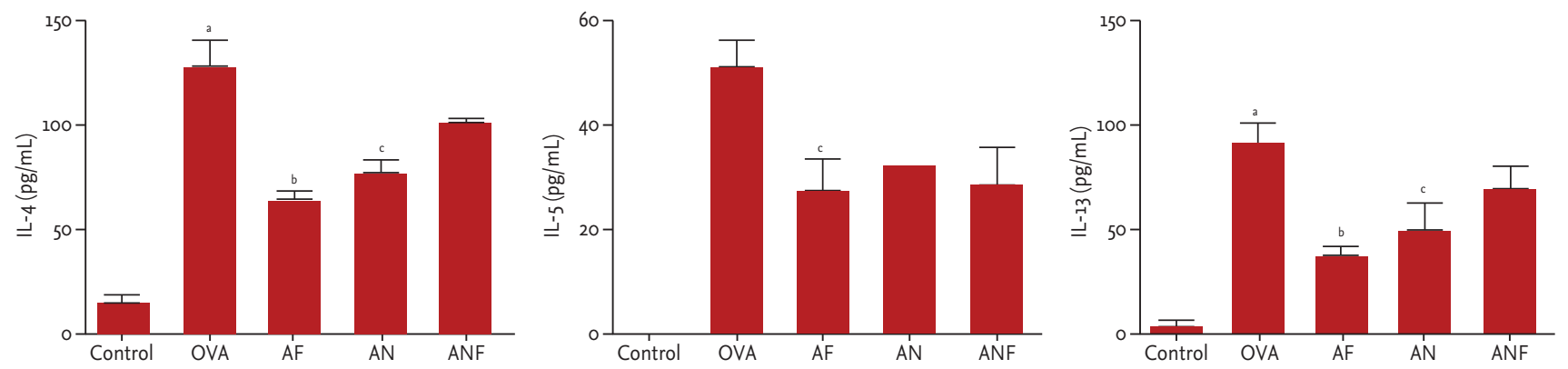

Figure 4. Effects of fluticasone and/or nilotinib on cytokine levels in bronchoalveolar lavage fluid. Values are expressed as mean $\pm \mathrm{SE}$ ( $\mathrm{n}=5$ to 9 per group). IL, interleukin; OVA, ovalbumin; AF, fluticasone; AN, nilotinib; ANF, fluticasone plus nilotinib. ${ }^{a} p<0.01$ compared with the control group, ${ }^{b} p<0.01,{ }^{c} p<0.05$ compared with the OVA group.

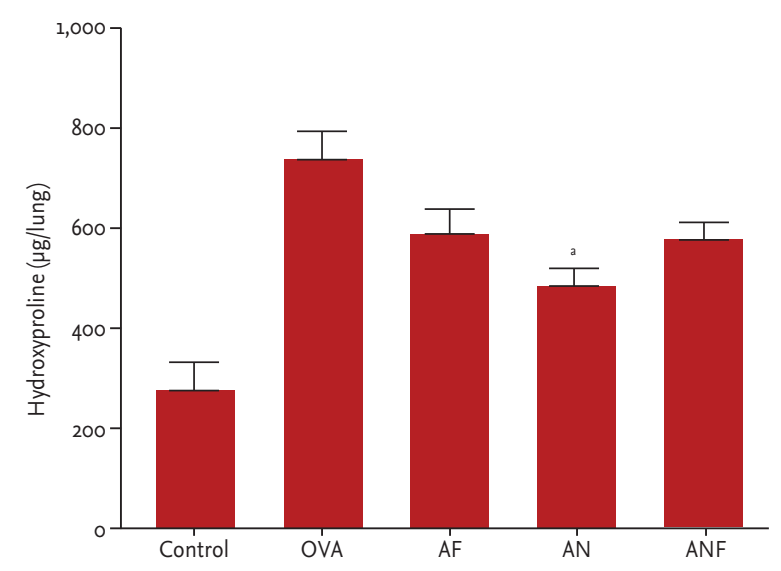

Figure 5. Effects of fluticasone and/or nilotinib on total lung collagen levels. Values are expressed as mean $\pm \mathrm{SE}(\mathrm{n}=5$ to 9 per group). OVA, ovalbumin; AF, fluticasone; AN, nilotinib; ANF, fluticasone plus nilotinib. ${ }^{a} p<0.05$ compared with the OVA group.

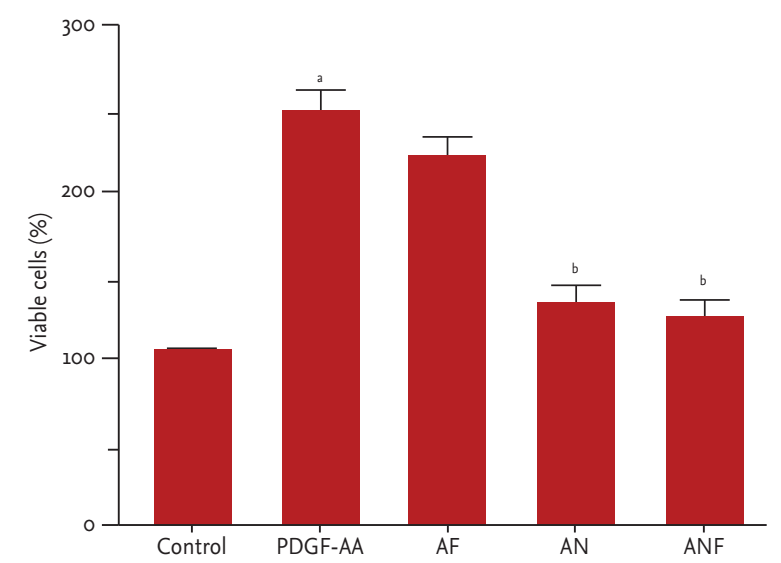

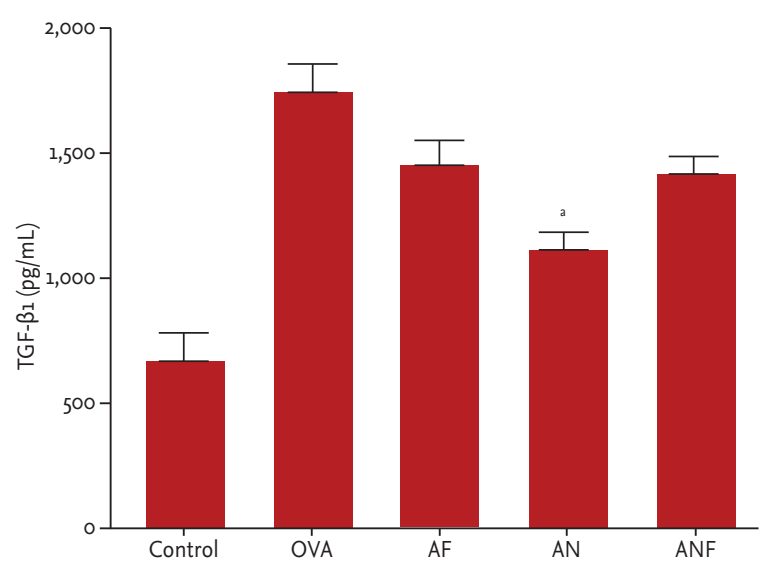

Figure 6. Effects of fluticasone and/or nilotinib on transforming growth factor (TGF)- $\beta 1$. Only nilotinib treatment significantly lowered the expression of TGF- $\beta 1$ compared with the ovalbumin (OVA) group. Values are expressed as mean $\pm \mathrm{SE}$ ( $\mathrm{n}=5$ to 9 per group). $\mathrm{AF}$, fluticasone; $\mathrm{AN}$, nilotinib; ANF, fluticasone plus nilotinib. ${ }^{a} p<0.05$ compared with the OVA group.

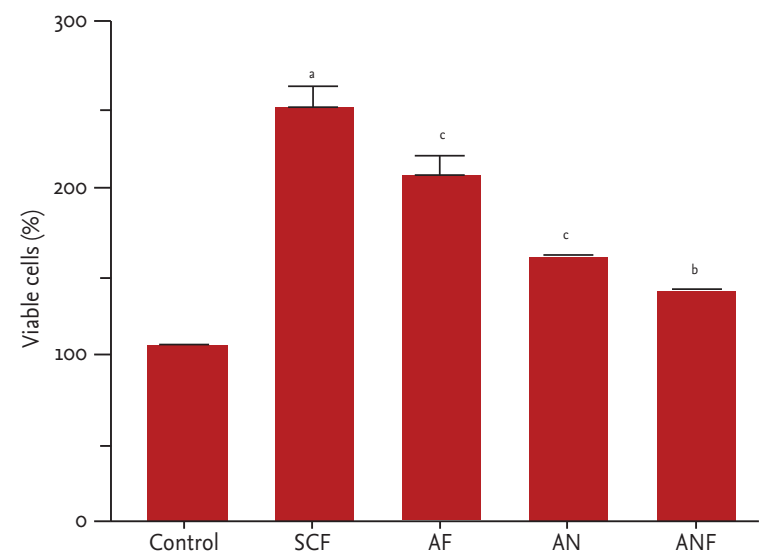

Figure 7. Effects of fluticasone and/or nilotinib on the growth of lung fibroblasts stimulated with platelet-derived growth factor (PDGF)-AA or stem cell factor (SCF). Cell proliferation induced by PDGF-AA was inhibited significantly by nilotinib treatment, but not fluticasone. SCF induced cell proliferation was inhibited by both nilotinib and fluticasone treatment, showing a synergistic effect. AF, fluticasone; AN, nilotinib; ANF, fluticasone plus nilotinib. ${ }^{a} p<0.01$ compared with the control group, ${ }^{b} p<$ $0.01,{ }^{c} p<0.05$ compared with the PDGF-AA or SCF group. 
choconstriction results in the release of growth factors, such as early growth response-1 and TGF- $\beta 1$ [17-21]. Our results support these findings in that inhaled corticosteroids did not affect collagen deposition or the level of TGF- $\beta 1$. Thus, novel therapeutic agents targeting airway fibrosis may offer added benefit to inhaled corticosteroids for preventing airway remodeling.

SCF, the ligand for the c-kit receptor, is expressed by inflammatory cells in the airways [22]. SCF is an important growth factor for mast cells, so binding of SCF and c-kit activates dysregulated mast cell-related disorders, including asthma. Thus, tyrosine kinase inhibitors targeting c-kit activity have been investigated in asthma [23]. PDGF is a chemoattractant for the migration of human airway smooth muscle cells, contributing to airway remodeling in asthma [24]. Tyrosine kinase inhibitors block the tyrosine kinase activity of PDGF receptors and thereby can prevent PDGF-induced PDGFR autophosphorylation and signaling [13]. Berlin et al. [25] showed that imatinib, the most widely recognized tyrosine kinase inhibitor, has a significant effect on chronic peribronchial allergen-induced fibrotic remodeling. Additionally, tyrosine kinase inhibitors simultaneously target two major profibrotic pathways, TGF- $\beta$ and PDGF signaling [26]. We previously showed that c-kit and PDGFR tyrosine kinase inhibitors such as imatinib and nilotinib attenuated airway remodeling in a chronic asthma model $[10,11]$. In the present study, nilotinib treatment significantly decreased collagen deposition. To investigate the inhibitory effect of nilotinib treatment on fibrotic remodeling, we assessed the growth of lung fibroblasts stimulated by PDGF and SCF in vitro. Treatment with nilotinib inhibited this cell proliferation significantly. We confirmed that the anti-fibrotic effect of nilotinib was mediated by inhibition of the c-kit and PDGFR pathways.

TGF- $\beta$ is a profibrotic cytokine, and TGF- $\beta$ isoforms have several roles in the regulation of airway inflammation and the remodeling process in asthma [27]. The levels of TGF- $\beta$ are increased in the airways in asthma and elevated further in response to allergen exposure [28]. TGF- $\beta$ stimulates the differentiation of fibroblasts to myofibroblast cells and their proliferation, resulting in extracellular matrix proteins [29,30]. TGF- $\beta$ also enhances airway smooth muscle proliferation, mediated by extracellular matrix-integrin interactions [31,32]. Thus, modulation of TGF- $\beta$ represents a potential therapeutic target for the prevention or reversal of airway remodeling [33]. Some studies have shown that nilotinib decreases the level of TGF- $\beta$. Liu et al. [34] suggested that nilotinib treatment inhibited not only PDGF and Abl signaling but also TGF- $\beta$ cytokines in vitro. In a murine lung injury model, TGF- $\beta$ expression was decreased by nilotinib treatment [15]. The results of the present study correspond well with these results. Nilotinib effectively attenuated airway smooth muscle hyperplasia and collagen deposition, decreasing levels of TGF- $\beta 1$.

Currently, treatment of asthma is focused on amelioration of inflammation and the reversal of bronchoconstriction using inhaled corticosteroids. However, treating only the inflammatory component using inhaled corticosteroids without controlling airway caliber does not prevent airway remodeling, resulting in the loss of lung function [35]. Although several clinical studies suggest that inhaled corticosteroids can inhibit airway remodeling, their effectiveness in reversing established remodeling is limited [7,35,36]. Furthermore, there is no effective therapeutic agent known to prevent or reverse airway remodeling, although the reversibility of airway remodeling has been suggested by studies conducted in animal models [37]. Given this, nilotinib may be a promising agent to reverse airway remodeling in asthma. Although our study did not show a synergistic effect of nilotinib and fluticasone in airway remodeling, each agent had therapeutic effects in remodeling through anti-inflammatory and anti-fibrotic pathways, respectively. The combination of nilotinib and inhaled corticosteroids may be a therapeutic option to reverse airway remodeling in asthma patients. Further study is needed to determine any synergistic effect of these agents, and a larger-scale clinical trial is required for asthma patients resistant to steroid treatment.

In conclusion, we demonstrated that fluticasone and nilotinib treatment inhibited smooth muscle thickening in relation to airway remodeling in a chronic asthma model. Only nilotinib reduced subepithelial fibrosis, through inhibition of PDGFR and c-kit. Our results provide evidence for the therapeutic potential of nilotinib in the treatment of chronic asthma resistant to steroid treatment. Further studies regarding the effect of nilotinib on airway remodeling are needed. 


\section{KEY MESSAGE}

1. Fluticasone and nilotinib suppressed airway remodeling in a chronic asthma model through anti-inflammatory and anti-fibrotic pathways, respectively.

2. There was little synergistic effect when combining fluticasone and nilotinib.

\section{Conflict of interest}

No potential conflict of interest relevant to this article was reported.

\section{REFERENCES}

1. Sumi Y, Hamid Q. Airway remodeling in asthma. Allergol Int 2007:56:341-348.

2. Prakash YS. Airway smooth muscle in airway reactivity and remodeling: what have we learned? Am J Physiol Lung Cell Mol Physiol 2013;305:L912-L933.

3. Manuyakorn W, Howarth PH, Holgate ST. Airway remodelling in asthma and novel therapy. Asian Pac J Allergy Immunol 2013;31:3-10.

4. Chetta A, Zanini A, Foresi A, et al. Vascular component of airway remodeling in asthma is reduced by high dose of fluticasone. Am J Respir Crit Care Med 2003;167:751-757.

5. Lee SY, Kim JS, Lee JM, et al. Inhaled corticosteroid prevents the thickening of airway smooth muscle in murine model of chronic asthma. Pulm Pharmacol Ther 2008;21:14-19.

6. Yick CY, Zwinderman AH, Kunst PW, et al. Glucocorticoid-induced changes in gene expression of airway smooth muscle in patients with asthma. Am J Respir Crit Care Med 2013;187:1076-1084.

7. Bergeron C, Hauber HP, Gotfried M, et al. Evidence of remodeling in peripheral airways of patients with mild to moderate asthma: effect of hydrofluoroalkane-flunisolide. J Allergy Clin Immunol 2005;116:983-989.

8. Chakir J, Shannon J, Molet S, et al. Airway remodeling-associated mediators in moderate to severe asthma: effect of steroids on TGF-beta, IL-11, IL-17, and type I and type III collagen expression. J Allergy Clin Immunol 2003;111:1293-1298.

9. Royce SG, Sedjahtera A, Samuel CS, Tang ML. Combina- tion therapy with relaxin and methylprednisolone augments the effects of either treatment alone in inhibiting subepithelial fibrosis in an experimental model of allergic airways disease. Clin Sci (Lond) 2013;124:41-51.

10. Rhee CK, Kim JW, Park CK, et al. Effect of imatinib on airway smooth muscle thickening in a murine model of chronic asthma. Int Arch Allergy Immunol 2011;155:243251.

11. Rhee CK, Kang JY, Park CK, et al. Effect of nilotinib on airway remodeling in a murine model of chronic asthma. Exp Lung Res 2014;40:199-210.

12. Ostendorf BN, le Coutre P, Kim TD, Quintas-Cardama A. Nilotinib. Recent Results Cancer Res 2014;201:67-80.

13. Distler JH, Distler O. Intracellular tyrosine kinases as novel targets for anti-fibrotic therapy in systemic sclerosis. Rheumatology (Oxford) 2008;47 Suppl 5:v10-v11.

14. Gordon JK, Spiera RF. Targeting tyrosine kinases: a novel therapeutic strategy for systemic sclerosis. Curr Opin Rheumatol 2010;22:690-695.

15. Rhee CK, Lee SH, Yoon HK, et al. Effect of nilotinib on bleomycin-induced acute lung injury and pulmonary fibrosis in mice. Respiration 2011;82:273-287.

16. Shiha GE, Abu-Elsaad NM, Zalata KR, Ibrahim TM. Tracking anti-fibrotic pathways of nilotinib and imatinib in experimentally induced liver fibrosis: an insight. Clin Exp Pharmacol Physiol 2014;41:788-797.

17. Al-Alawi M, Hassan T, Chotirmall SH. Transforming growth factor $\beta$ and severe asthma: a perfect storm. Respir Med 2014;108:1409-1423.

18. Descalzi D, Folli C, Nicolini G, et al. Anti-proliferative and anti-remodelling effect of beclomethasone dipropionate, formoterol and salbutamol alone or in combination in primary human bronchial fibroblasts. Allergy 2008;63:432437.

19. Tschumperlin DJ, Drazen JM. Mechanical stimuli to airway remodeling. Am J Respir Crit Care Med 2001;164(10 Pt 2):S9o-S94.

20. Ressler B, Lee RT, Randell SH, Drazen JM, Kamm RD. Molecular responses of rat tracheal epithelial cells to transmembrane pressure. Am J Physiol Lung Cell Mol Physiol 2000;278:L1264-L1272.

21. Grainge CL, Lau LC, Ward JA, et al. Effect of bronchoconstriction on airway remodeling in asthma. N Engl J Med 2011;364:2006-2015.

22. Reber L, Da Silva CA, Frossard N. Stem cell factor and its receptor c-Kit as targets for inflammatory diseases. Eur J 
Pharmacol 2006;533:327-340.

23. El-Agamy DS. Targeting c-kit in the therapy of mast cell disorders: current update. Eur J Pharmacol 2012;690:1-3.

24. Carlin SM, Roth M, Black JL. Urokinase potentiates PDGF-induced chemotaxis of human airway smooth muscle cells. Am J Physiol Lung Cell Mol Physiol 2003; 284:L1020-L1026.

25. Berlin AA, Hogaboam CM, Lukacs NW. Inhibition of SCF attenuates peribronchial remodeling in chronic cockroach allergen-induced asthma. Lab Invest 2006;86:557565 .

26. Distler JH, Distler O. Tyrosine kinase inhibitors for the treatment of fibrotic diseases such as systemic sclerosis: towards molecular targeted therapies. Ann Rheum Dis 2010;69 Suppl 1:i48-i51.

27. Bottoms SE, Howell JE, Reinhardt AK, Evans IC, McAnulty RJ. Tgf-Beta isoform specific regulation of airway inflammation and remodelling in a murine model of asthma. PLoS One 2010;5:e9674.

28. Redington AE, Madden J, Frew AJ, et al. Transforming growth factor-beta 1 in asthma. Measurement in bronchoalveolar lavage fluid. Am J Respir Crit Care Med 1997;156(2 Pt 1):642-647.

29. Michalik M, Pierzchalska M, Legutko A, et al. Asthmatic bronchial fibroblasts demonstrate enhanced potential to differentiate into myofibroblasts in culture. Med Sci Monit 2009;15:BR194-BR201.

30. Kumawat K, Menzen MH, Bos IS, et al. Noncanonical
WNT-5A signaling regulates TGF- $\beta$-induced extracellular matrix production by airway smooth muscle cells. FASEB J 2013;27:1631-1643.

31. Oenema TA, Mensink G, Smedinga L, et al. Cross-talk between transforming growth factor- $\beta_{1}$ and muscarinic $M_{2}$ receptors augments airway smooth muscle proliferation. Am J Respir Cell Mol Biol 2013;49:18-27.

32. Makinde T, Murphy RF, Agrawal DK. The regulatory role of TGF-beta in airway remodeling in asthma. Immunol Cell Biol 2007;85:348-356.

33. Howell JE, McAnulty RJ. TGF-beta: its role in asthma and therapeutic potential. Curr Drug Targets 2006;7:547-565.

34. Liu Y, Wang Z, Kwong SQ, et al. Inhibition of PDGF, TGF- $\beta$, and Abl signaling and reduction of liver fibrosis by the small molecule Bcr-Abl tyrosine kinase antagonist Nilotinib. J Hepatol 2011;55:612-625.

35. Vanacker NJ, Palmans E, Kips JC, Pauwels RA. Fluticasone inhibits but does not reverse allergen-induced structural airway changes. Am J Respir Crit Care Med 2001;163(3 Pt 1):674-679.

36. Olivieri D, Chetta A, Del Donno M, et al. Effect of shortterm treatment with low-dose inhaled fluticasone propionate on airway inflammation and remodeling in mild asthma: a placebo-controlled study. Am J Respir Crit Care Med 1997;155:1864-1871.

37. Hirota N, Martin JG. Mechanisms of airway remodeling. Chest 2013;144:1026-1032. 